\title{
Review of: "Longitudinal detection of somatic mutations in saliva and plasma for the surveillance of oral squamous cell carcinomas"
}

\author{
tony Godfrey ${ }^{1}$, Gordana rasic ${ }^{1}$, Anand Devaiah ${ }^{1}$
}

1 Boston University

Potential competing interests: The author(s) declared that no potential competing interests exist.

\begin{abstract}
Longitudinal detection of somatic mutations in saliva and plasma for the surveillance of oral squamous cell carcinomas Invited Commentary
\end{abstract}

Authors: Gordana Rasic, MD MS; Anand Devaiah, MD; Tony E. Godfrey, PhD

In this study, Cui and colleagues developed novel methodology to detect circulating tumor DNA (ctDNA) in both plasma and saliva samples of patients with oral squamous cell carcinoma and assessed the efficacy of ctDNA monitoring for postoperative surveillance.(1) In total, eleven patients with varying stages of resectable disease were recruited and 77 of 88 anticipated samples were collected, reflecting consistent patient follow-up. Results revealed earlier detection of recurrence by ctDNA monitoring when compared to standard surveillance methods - history, exam, and imaging - with an average 4.4 month lead time. Saliva ctDNA was found to be more sensitive for detection of recurrence when compared to plasma ctDNA. When used collectively, saliva and plasma ctDNA demonstrated the highest detection sensitivity for recurrence detection ( 5 of 6 patients; 83.33\%). This study suggests that while both saliva and plasma are possible specimens for liquid biopsy in oral squamous cell carcinoma, salivary samples may be the more desired approach for loco-regional recurrence detection.

In head and neck cancer, ctDNA has the potential to overcome diagnostic challenges during initial assessment, treatment response monitoring, and postoperative surveillance. Both saliva and plasma have been identified as promising specimens for ctDNA analysis, however it is uncertain if either specimen or the combination of the two specimens is superior for clinical monitoring. To address this question, two factors regarding the sample source must be taken into consideration - 1) the anatomic location of the cancer and 2) the quality of the patient sample.

This study suggests that saliva ctDNA is more sensitive for loco-regional recurrence in oral cavity cancers when compared to plasma ctDNA, but little information is provided about its sensitivity for disease outside of the oropharynx - in terms of recurrence and new primary lesions. A recent study by Wang et al. evaluated 93 patients with squamous cell carcinoma of the head and neck and found that while saliva 
ctDNA was more sensitive for loco-regional oral cavity cancers, plasma ctDNA had higher detection rates for distant metastases. (2) In addition, plasma ctDNA was found to have higher sensitivity for non-oral head and neck cancers. Perdomo et al. described similar trends where ctDNA analysis of oral rinses exhibited worse sensitivity for sites outside of the oral cavity.(3) Based on these studies, while saliva ctDNA demonstrates higher resolution for local disease of oral cavity cancers, plasma ctDNA better captures distant disease for both primary and secondary tumors, emphasizing its continued importance in both oral and non-oral cancers.

The quality of the patient sample also requires important consideration when determining the specimen source for ctDNA analysis. Saliva samples require less invasive approaches for acquisition when compared to plasma samples. However, head and neck cancer patients are exposed to treatments that may impede their ability to provide sufficient -if any- amount of saliva. Xerostomia is a common sequela of radiation therapy to the oral cavity and neck, and unfortunately, it has no causal treatment other than symptomatic relief. (4) Salivary stimulants can be employed to improve the specimen amount collected, but it does not guarantee that the appropriate amount of ctDNA will be isolated from the sample. As a result, plasma samples offer a more reliable biological specimen for patients with head and neck cancer and should remain as a potential biologic specimen source for perioperative surveillance.

In terms of methodology, mutations in ctDNA can be detected with either personalized panels or large, cancer-specific panels. Personalized panels are carefully tailored to detect a small number of mutations specific to each patient while cancer-specific panels target hundreds or thousands of genes that are commonly mutated in the given tumor type. As a result, large panels cast a wide net for capturing tumor-specific mutations that allow for subsequent monitoring of tumor heterogeneity and clonal evolution during treatment, but large panels are associated with longer turn-around-time, higher complexity and cost, and potentially lower sensitivity for recurrence detection versus a personalized panel.(5) These factors need to be considered when choosing an appropriate ctDNA detection method in future studies and clinical trials.

In summary, head and neck cancers present diagnostic and therapeutic challenges, even in terms of the methodology of ctDNA monitoring. Future research efforts should continue to evaluate the efficacy of ctDNA in postoperative surveillance of head and neck cancers as well as to identify the optimal specimen for each cancer type and stage. Lastly, future directions should aim to identify methodologies that enable sensitive ctDNA detection for cancer monitoring along with cost-effectiveness and institutional accessibility.

\section{References:}

1. Cui Y, Kim H-S, Cho ES, et al. Longitudinal detection of somatic mutations in saliva and plasma for the surveillance of oral squamous cell carcinomas. PLoS One. 2021;16(9):e0256979.

2. Wang Y, Springer S, Mulvey CL, et al. Detection of somatic mutations and HPV in the saliva and plasma of patients with head and neck squamous cell carcinomas. Sci Transl Med. 2015;7(293):293ra104. 
3. Perdomo S, Avogbe PH, Foll M, et al. Circulating tumor DNA detection in head and neck cancer: evaluation of two different detection approaches. Oncotarget. 2017;8(42):72621-72632.

4. Guchelaar, HJ., Vermes, A. \& Meerwaldt, J. Radiation-induced xerostomia: pathophysiology, clinical course and supportive treatment. Support Care Cancer 5, 281-288 (1997). https://doi.org/10.1007/s005200050075

5. Keller L, Belloum Y, Wikman H, Pantel K. Clinical relevance of blood-based ctDNA analysis: mutation detection and beyond. BrJ Cancer. 2021;124(2):345-358. 